\title{
Monitoring Change Through Hierarchical Segmentation of Remotely Sensed Image Data
}

\author{
James C. Tilton \\ Mail Code 606 \\ NASA's Goddard Space Flight Center \\ Greenbelt, MD 20771 \\ James.C.Tilton@nasa.gov
}

\author{
William T. Lawrence \\ Natural Sciences \\ Bowie State University \\ Bowie, MD 20715 \\ wlawrence@bowiestate.edu
}

\begin{abstract}
NASA's Goddard Space Flight Center has developed a fast and effective method for generating image segmentation hierarchies. These segmentation hierarchies organize image data in a manner that makes their information content more accessible for analysis. Image segmentation enables analysis through the examination of image regions rather than individual image pixels. In addition, the segmentation hierarchy provides additional analysis clues through the tracing of the behavior of image region characteristics at several levels of segmentation detail. The potential for extracting the information content from imagery data based on segmentation hierarchies has not been fully explored for the benefit of the Earth and space science communities. This paper explores the potential of exploiting these segmentation hierarchies for the analysis of multi-date data sets, and for the particular application of change monitoring.
\end{abstract}

Keywords-hierarchical segmentation; MODIS; change detection; monitoring

\section{INTRODUCTION}

Image segmentation is the partitioning of an image into related sections or regions. For remotely sensed images of the earth, an example of an image segmentation would be a labeled map that divides the image into areas covered by distinct earth surface covers such as water, snow, types of natural vegetation, types of rock formations, types of agricultural crops and types of other man created development. In unsupervised image segmentation, the labeled map may consist of generic labels such as region 1 , region 2 , etc., which may be converted to meaningful labels by a post-segmentation analysis.

Segmentation is a key first step for a number of approaches to image analysis and compression. In image analysis, the group of image points contained in each region provides a good statistical sampling of image values for more reliable labeling based on region mean feature values. In addition, the region shape can be analyzed for additional clues to the appropriate labeling of the region. In image compression, the regions form a basis for compact representation of the image. The quality of the prerequisite image segmentation is a key factor in determining the level of performance for these image analysis and compression approaches.

A segmentation hierarchy is a set of several segmentations of the same image at different levels of detail in which the segmentations at coarser levels of detail can be produced from simple merges of regions at finer levels of detail. This is useful for applications that require different levels of image segmentation detail depending on the particular image objects segmented. A unique feature of a segmentation hierarchy that distinguishes it from most other multilevel representations is that the segment or region boundaries are maintained at the full image spatial resolution for all levels of the segmentation hierarchy.

In a segmentation hierarchy, an object of interest may be represented by multiple image segments in finer levels of detail in the segmentation hierarchy, and may be merged into a surrounding region at coarser levels of detail in the segmentation hierarchy. If the segmentation hierarchy has sufficient resolution, the object of interest will be represented as a single region segment at some intermediate level of segmentation detail. The segmentation hierarchy may be analyzed to identify the hierarchical level at which the object of interest is represented by a single region segment. The object may then be identified through its spectral and spatial characteristics. Additional clues for object identification may be obtained from the behavior of the image segmentations at the hierarchical segmentation levels above and below the level at which the object of interest is represented by a single region.

Segmentation hierarchies may be formed through a region growing approach to image segmentation. In region growing, spatially adjacent regions iteratively merge through a specified merge selection process. Hierarchical Step-Wise Optimization (HSWO) is a form of region growing segmentation in which the iterations consist of finding the best segmentation with one region less than the current segmentation [1], [2]. The best segmentation is defined through a mathematical criterion such as a minimum vector norm or minimum mean squared error. HSWO may be initialized from a pre-segmentation provided by another segmentation approach, or may be initialized from single pixel regions. An augmentation of HSWO, called HSEG (for Hierarchical Segmentation), was introduced in [3] in which spatially non-adjacent regions are allowed to merge controlled by a threshold based on previous merges of spatially adjacent regions. HSEG also includes a method for selecting the most "significant" iterations from which the segmentation result is saved into the output segmentation hierarchy. A recursive, divide-and-conquer, approximation of HSEG, called RHSEG, was first introduced in [4] and further described in [5]. A parallel implementation of RHSEG is given in [6]. The latest 
refinements of HSEG and RHSEG are detailed in [7] and [8]. The current implementation of RHSEG is so efficient that a full Landsat Thematic Mapper (TM) scene (roughly 7000 by 6500 pixels) can be processed in 5 to 10 minutes (depending on parameter settings) on a Beowulf cluster consisting of 256 2.4GHz CPUs (http://thunderhead.gsfc nasa.gov). This is only 10 to 20 times the amount of time the Landsat TM sensor takes to collect this amount of data.

The current state of change detection/environmental monitoring tends to use data sets collected with either high temporal frequency and moderate spatial resolution, or limited observations at high spatial resolution. This is, for the most part, a simple artifact of the revisit time of the sensors or the spatial resolution required for detection of the phenomenon under study. A good example of the former is NOAA NESDIS Automated Biomass Burning Algorithm [ABBA http://www.ssd.noaa.gov/PS/FIRE/hms.html] where wild fires detected with the NOAA Advanced Very High Resolution Radiometer [AVHRR] and posted in near-real-time to their Web site for the user community. An example of the latter approach would be a monitoring program like the Land Cover Mapping and Monitoring Program (LCMMP) of the USDA Forest Service (FS) and the California Department of Forestry and Fire Protection (CDF) [http://www.fs.fed.us/r5/spf/about/ fhp-change.shtml]. Their program carries out vegetation mapping and long-term monitoring using high spatial resolution remotely sensed data. Not only extent, but condition and cause in the case of change in land cover, are posted.

As the availability of frequently acquired satellite data sets for Earth science applications is burgeoning, we see an opportunity for its utilization. With the near-term advent of the National Polar-orbiting Operational Environmental Satellite System [NPOESS] and the NPOESS Preparatory Project Mission [NPP] even more data will become available. Currently, beside archival data, satellite instrument data sets are available in real-time through direct broadcast or in near-realtime through a number of venues. These data are available for analysis within a few minutes, or at most a few hours, from acquisition. This immediacy of access calls for immediacy of analysis and potential action when used for environmental, security, hazards monitoring. In fact many programs are promoting global change monitoring strategies that would use frequent acquisitions and analysis [9]. This project will focus on change monitoring using near-real-time data in a continuous mode, not at a yearly, multi-year or decadal scale using composite data sets. Due to the potentially large amounts and frequency of data, an automated system is critical for its success.

In the next section we describe a collection of MODIS we have assembled for our experiments. After that we present some preliminary analysis results from processing these MODIS data sets with RHSEG and outline our planes for additional analysis.

\section{MODIS DATA SETS ASSEMBLED FOR THIS STUDY}

\section{A. Acquisition of Data Sets}

MODIS data is available from a number of sources, but we used the NASA Goddard Space Flight Center Data and Information Services Center [DISC] Distributed Active Archive Center [DAAC] as our source. Since we were seeking multi-date MODIS granules, we use the MODIS Multiple Data Ordering Page [MDOP] web interface. [Data Access, MODIS Multiple Data through http://daac.gsfc.nasa.gov/MODIS]. The interface allows spatial searching with a 'rubber band' on map or geographic coordinate box. The site offers a wide range of MODIS radiometric, geolocation, atmosphere and oceans products. A channel subset function is available for some $1 \mathrm{KM}$ data sets. Recent data is available through immediate FTP, while archived data is staged for push or pull FTP from the library and users advised by email of data availability for download.

\section{B. Data Sets Used in this Study}

We used MODIS Terra MOD021KM calibrated at aperture radiances and MOD03 geolocation data. From the MOD021KM data, we acquired only the reflected bands $1-7$, a feature of the MDOP system. Since this was a study of change detection, we acquired seasonal data sets from early 2003 through early 2005. The acquisitions were meant to capture both seasonal change, and as a test of change detection, large chaparral fires that occurred in Southern California in fall 2003. We bounded our search with the geographic coordinate spatial search, using $32^{\circ} \mathrm{N}, 30^{\circ} \mathrm{N}, 117^{\circ} \mathrm{W}$ and $116^{\circ} \mathrm{W}$ as our limits. We collected 12 MODIS granules for analysis. Our selection criteria included seasonality and cloud cover over the Southern California region. Once data orders were made, the data granules were usually available over night for FTP pull. Data sets are uncompressed, distributed in $\mathrm{HDF}$.

TABLE I. MODIS DATA SETS USED

\begin{tabular}{|l|c|c|}
\hline \multicolumn{3}{|c|}{ MOD03 \& MODO21 KM Data Granules } \\
\hline Granule name & Date & Notes \\
\hline A2003031.1815 & 31 JAN 2003 & Winter \\
\hline A2003221.1825 & 19 APR 2003 & Spring \\
\hline A2003294.1820 & 21 AUG 2003 & Summer \\
\hline A2003301.1825 & 28 OCT 2003 & Pre-fire \\
\hline A2003322.1845 & 18 NOV 2003 & Post-fire \\
\hline A2004032.1825 & 01 FEB 2004 & Winter \\
\hline A2004080.1825 & 20 MAR 2004 & Spring \\
\hline A2004163.1855 & 11 JUN 2004 & Summer \\
\hline A2004268.1850 & 24 SEP 2004 & Summer \\
\hline A2004334.1835 & 29 NOV 2004 & Winter \\
\hline A2005059.1820 & 28 FEB 2005 & Spring \\
\hline
\end{tabular}

a. only used granules from Terra, AM1 platform 


\section{MODIS Data Processing}

Prior to image segmentation and change analysis the seven reflective band MODIS data were converted from swath to georectified grid, from HDF to binary BSQ, normalized difference vegetation index calculated and added as an eighth band, and then a uniform geographic subset made so that all 12 data sets were coincident. The final data sets were approximately 1000 X $1000 \mathrm{~km}$ with a $1 \mathrm{~km}$ spatial resolution. Data were moved to our local network attached storage device using a freeware GUI-based Windows FTP client program [FileZilla, filezilla.sourceforge.net]. Further processing was carried out with ENVI [the Environment for Visualizing Images, Research Systems, Inc., Boulder, CO].

\section{PRELIMINARY ANALYSIS WITH RHSEG}

\section{A. Land/Water Mask Generation}

We will be primarily interested in monitoring changes in land cover. Therefore, an initial analysis task is to generate a land/water mask. Another reason for generating a land/water mask is that such a mask makes a cloud mask much easier, since clouds can look spectrally different over water than they look over land. However, a quick look at all twelve MODIS data sets shows that not one data set is completely cloud free. This complicates the task of generating a land/water mask.

The most cloud free data set is A2004334.1835, which is displayed in Fig.1. The coarsest segmentation from the segmentation hierarchy produced by RHSEG produces a two region segmentation in which the two regions correspond primarily to water and to land. However, there are a few small sub-regions in what should be ocean that are labeled as land, due to some small clouds over the ocean (lower center of Fig. 1). This confusion does not occur over land, since the two region segmentation places clouds with land.

This problem can be corrected by taking advantage of the transient nature of clouds, through the combining of the analysis of data set A2004334.1835 with other relatively cloudfree data sets. The next two most cloud-free data sets are A2003322.1845 and A2004268.1850, which are displayed in Fig. 2 and Fig. 3, respectively. Even though there is a large cloud bank in the lower left corner of Fig. 2, and ... of Fig. 3, these images are still useful in helping to determine the land/water mask since there is at least one image where the water areas are cloud-free.

The land/water mask can now be generated by designating as land only those areas that are in the "land" region for all three data sets (from the coarse two region segmentations produced by RHSEG). The result is shown in Fig. 4.

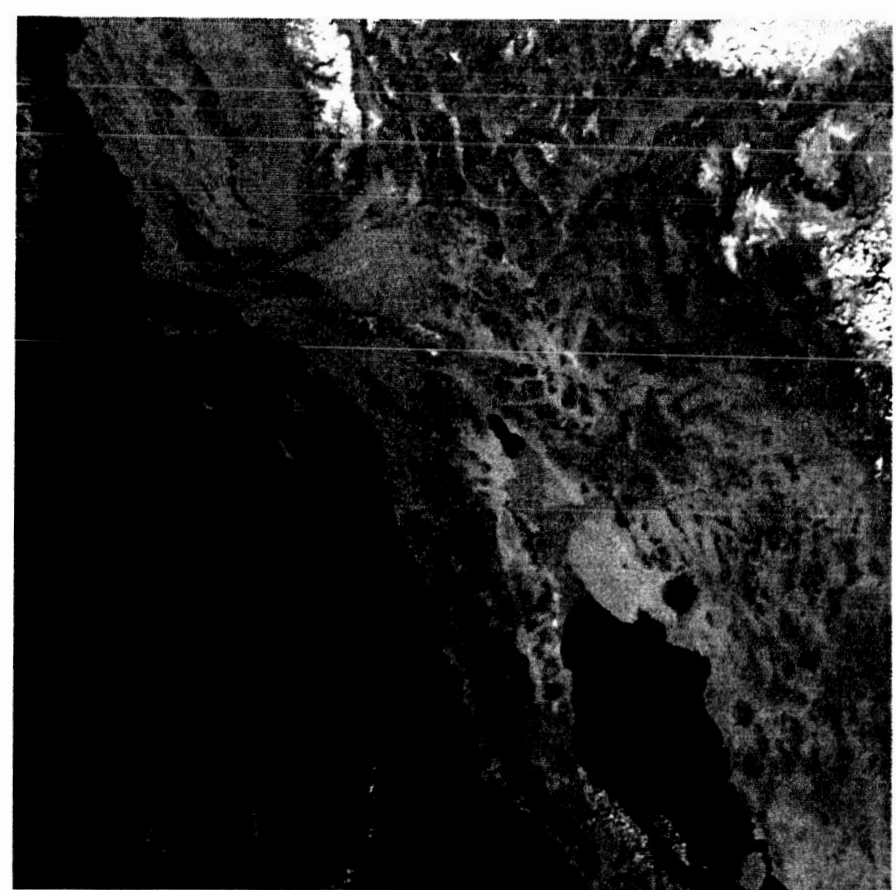

Figure 1. MODIS granule A2004334.1835, bands 3, 2 and 1 display as Red, Green and Blue. This is the most cloud-free data set of the twelve used in this study.

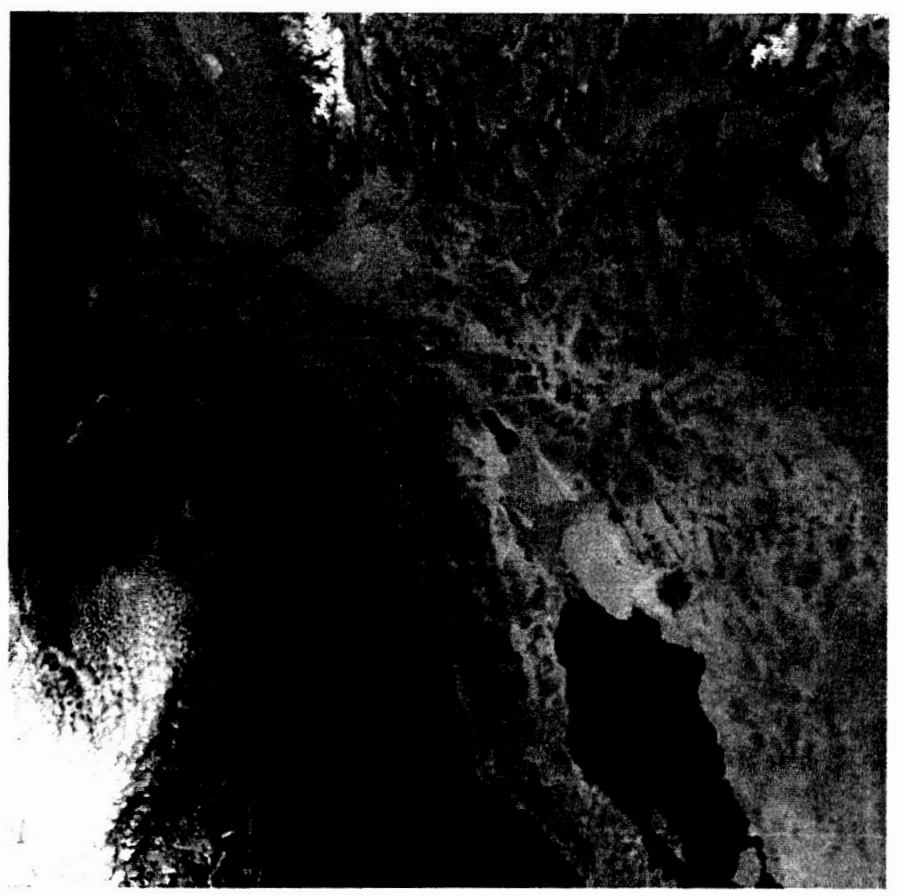

Figure 2. MODIS granule A2003322.1835, bands 3, 2 and 1 display as Red Green and Blue. This is one of the three data sets, along with Fig. 1 and Fig.3, used to generate a land/water mask. 


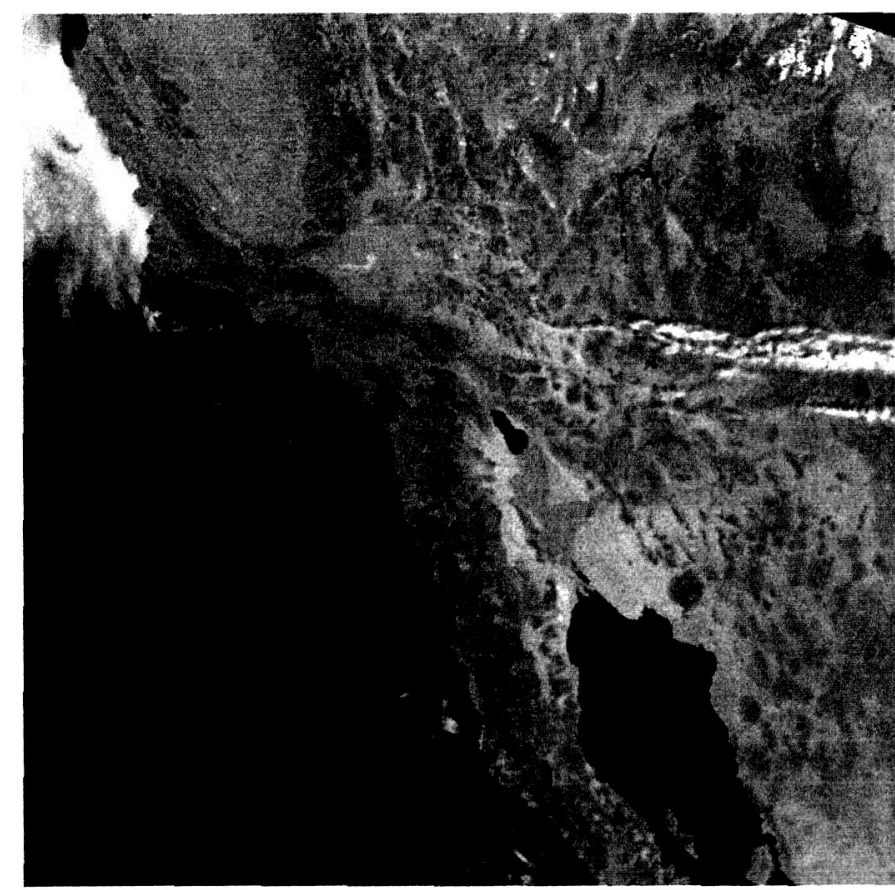

Figure 3. MODIS granule A2004268.1835, bands 3, 2 and 1 display as Red, Green and Blue. This is one of the three data sets, along with Fig. 1 and Fig.2, used to generate a land/water mask.

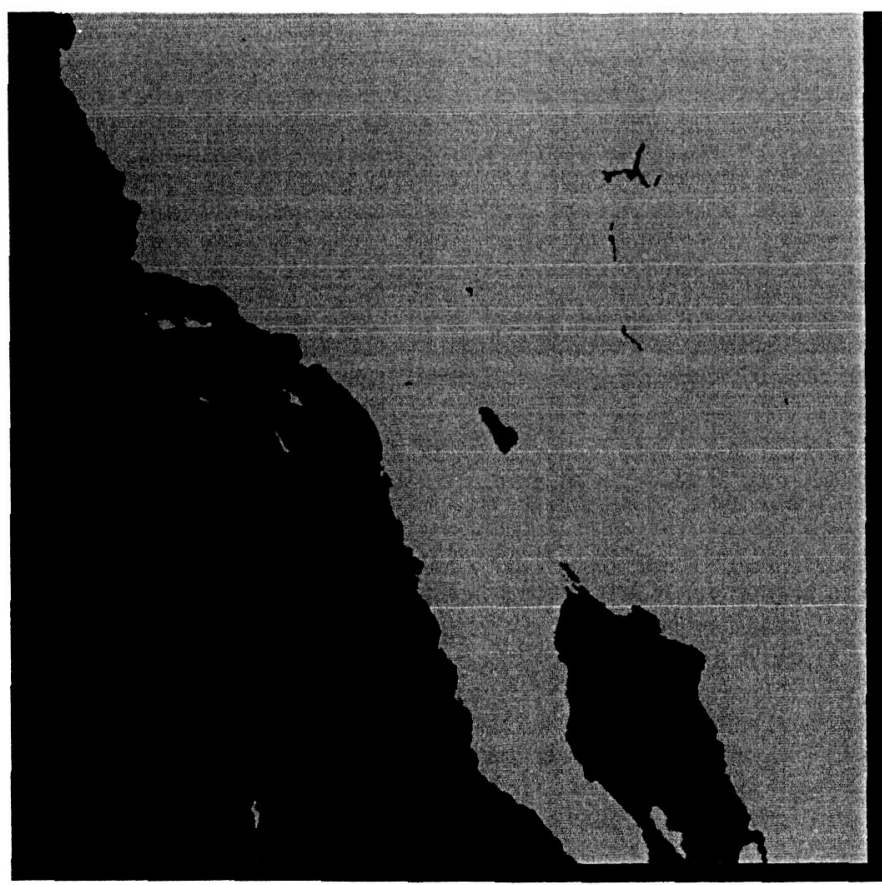

Figure 4. A land/water mask produce from combining the coarsest RHSEG segmentations from data sets A2004334.1835, A2003322.1835, and A2004268.1835.

\section{B. Cloud Mask Generation and Detection of other Bright Regions (over land)}

The Land/Water mask was applied to each data set, and RHSEG was used to process just the land image pixels. Information on selected regions from the segmentation hierarchy for each data set is given in TABLE II. The segmentation hierarchy was initially examined to see if there were any bright regions that were so dominate that they persisted all the way to the coarsest level of the segmentation hierarchy. This was the case only for MODIS granule A2003031.1815, where a 25,873 pixel cloudy area (region 25) persisted all the way up to the coarsest hierarchical level (level 35 ). Before additional analysis is performed on this data set, it would be probably advisable to mask out this dominate cloud region.

For the other data sets, the bright regions (clouds, snow or smoke) did not persist as separate regions up to the coarsest level of the segmentation hierarchy. As detailed in TABLE II, for MODIS granule A2003109.1825, a 50,054 pixel region that is apparently mountaintop snow persists all the way up to the second coarsest level of the segmentation hierarchy. For MODIS granule A2003221.1825, a small 7,524 pixel region of mountain ridge clouds also persists all the way up to the second coarsest level of the segmentation hierarchy. There are no bright regions in MODIS granule A2003294.1820. However, some cloud and smoke regions appear in MODIS granule A2003301.1825. Here region 34, which combines both clouds and smoke, persists to hierarchical 23 out of 27 . The smoke region can be separated out from the cloud region as region 35 at hierarchical level 8 . Two small mountain snow regions are seen in MODIS granule A2003322.1845. One is region 52 at hierarchical level 29 out of 32 and the other is region 55 at hierarchical level 23 out of 32 . A region combining clouds and snow (region 26) persists all the way up to the second coarsest level of the segmentation hierarchy in MODIS granule A2004032.1825. This region can be separated into constituent regions of snow and clouds at finer levels of the segmentation hierarchy (not shown in TABLE II). Three small bright regions appear in MODIS granule A2004080.1825. Region 44 at hierarchical level 29 out of 30 is mountain snow, region 48 at hierarchical level 26 covers cloudy areas along the coast, and region 45 at hierarchical level 23 is apparently mountaintop clouds. MODIS granule A2004163.1855 has two small cloudy regions, regions 56 and 55 , persisting up to hierarchical levels 30 and 29 , respectively, out of 35 hierarchical levels. Similarly, MODIS granule A2004268.1850 has two small regions that are apparently clouds, regions 49 and 59 , persisting up to hierarchical level 23 out of 28 hierarchical levels. A fairly large snow region of 14,318 pixels (region 31 ) persists up to the second coarsest hierarchical level in MODIS granule A2004334.1835. A smaller region (region 44) that is apparently clouds persists up to hierarchical level 26 out of 30 . Finally, a moderately large cloudy region (region 33) persists up to the second coarsest hierarchical level in MODIS granule A2004049.1820.

The above analysis of the RHSEG segmentation hierarchies was performed with a user-interactive analysis tool called HSEGViewer. This tool is available along with a time-limited demo version of the RHSEG program through the NASA Goddard Space Flight Center's Technology Transfer Office at http://techtransfer.gsfc.nasa.gov/RHSEG/. An automated approach for identifying bright regions in the segmentation hierarchies should be ready for discussion at the workshop. 


\section{PLANS FOR ADDITIONAL ANALYSIS}

More detailed studies of change will focus on changes at the landscape level such as those due to interannual variability in vegetation areal extent and status, variability in seasonality [onset of growth, drought conditions] or more acute, but short lived phenomenon like insect or weather-related damage, or longer term impacts like fire. The spectral coverage of MODIS even makes it possible to detect algal blooms, sediment plumes and other disturbance in water bodies. Within the two year period of the data collected for this project, examples can be found of all of these changes and more.

One of our major goals is to create an automated change detection algorithm that can not only flag areas of change for 'human' follow up, but that can determine if the change is 'important' or not. The definition of 'importance' would depend on the final use of the system, and the operational agency and its needs, but filtering is critical so that every 'change' doesn't create a flag. We envision filtering out ephemeral changes due to weather, short-term precipitation regimes or recognized seasonality. The use of long-term, but high frequency of acquisition data would allow training the change detection for effective filtering of potential false positives.

\section{REFERENCES}

[1] J. C. Tilton and S. C. Cox, "Segmentation of remotely sensed data using parallel region growing," 1983 International Geoscience and Remote Sensing Symposium (GGARSS'83) Digest, San Francisco, CA, Vol. 1, Section WP-4, paper 9, Aug. 31-Sept. 2, 1983.

[2] J-M. Beaulieu and M. Goldberg, "Hierarchy in picture segmentation: A stepwise optimal approach," IEEE Transactions on Pattern Analysis and Machine Intelligence, Vol. 11, No. 2, pp. 150-163, Feb. 1989.
[3] James C. Tilton, "Image Segmentation by Region Growing and Spectral Clustering with a Natural Convergence Criterion," Proceedings of the 1998 International Geoscience and Remote Sensing Symposium, Seattle, WA, pp. 1766-1768, July 6-10, 1998.

[4] J. C. Tilton, "A recursive PVM implementation of an image segmentation algorithm with performance comparisons in-between the HIVE and Cray T3E," Proceedings of the Seventh Symposium on the Frontiers of Massively Parallel Computation, Annapolis, MD, pp. 146153, Feb. 21-25, 1999.

[5] James C. Tilton, "Method for recursive hierarchical segmentation by region growing and spectral clustering with a natural convergence criterion," Disclosure of Invention and New Technology: NASA Case No. GSC 14,328-1, February 28, 2000. See also http://code 935 gsfonasa.gov/code935/tilton/index.html.

[6] J. C. Tilton, "Method for implementation of recursive hierarchical segmentation on parallel computers," Disclosure of Invention and New Technology (Including Software): NASA Case No. GSC 14,305-1, NASA's Goddard Space Flight Center, Feb. 2, 2000. NOTE: U. S. Patent Application Serial No. 09/839,147 was filed on this technology on April 23, 2001.

[7] James C. Tilton, "A split-remerge method for eliminating processing window artifacts in recursive hierarchical segmentation," Disclosure of Invention and New Technology: NASA Case No. GSC 14,994-1, March 9, 2005.

[8] James C. Tilton, "An innovative utilization of the heap data structure for efficient determination of best merges for hierarchical segmentation," Disclosure of Invention and New Technology: NASA Case No. GSC 14,995-1, March 9, 2005.

[9] US Climate Change Science Program, "Strategic Plan for the Climate Change Science Program. A report by the Climate Change Science Program and the Subcommittee on Global Change Research", Final Report, July 2003. US Climate Change Science Program, Suite 250, 1717 Pennsylvania Ave, NW, Washington, DC 20006. 
TABLE II. SELECTEd REgIONS FROM THE SEgMENTATION HIERARCHY FOR EACH DATA SET. (C) DESIGNATES CLOUDS, (Sn) DESIGNATES SNOW, AND (Sm) DESIGNATES SMOKE.

\begin{tabular}{|c|c|c|c|c|}
\hline Granule name & $\begin{array}{c}\text { Hierarchical } \\
\text { Level }\end{array}$ & $\begin{array}{c}\text { Region } \\
\text { Label }\end{array}$ & $\begin{array}{l}\text { Number } \\
\text { of Pixels }\end{array}$ & Mean Vector \\
\hline A2003031.1815 & 35 & $25(\mathrm{C})$ & 23873 & $(5499,9961,10703,9692,9231,8571,7166)$ \\
\hline "6 & 35 & 1 & 579708 & $(2057,4752,3544,3286,5073,5262,5081)$ \\
\hline A2003109.1825 & 33 & 3 & 308797 & $(4410,9260,6270,6608,9601,9970,10099)$ \\
\hline “ & 33 & 1 & 294784 & $(2332,6700,4137,3893,6914,6526,5777)$ \\
\hline “ & 32 & $32(\mathrm{Sn} ?)$ & 50054 & $(7216,13391,12150,12009,11739,9805,8307)$ \\
\hline A2003221.1825 & 29 & 2 & 284594 & $(4129,8682,5422,5938,9530,10537,10277)$ \\
\hline " & 29 & 1 & 318987 & $(2448,6574,3972,3878,7136,7230,6251)$ \\
\hline " & 28 & $41(\mathrm{C})$ & 7524 & $(9938,17035,16531,16601,14732,11293,8696)$ \\
\hline A2003294.1820 & 30 & 1 & 364576 & $(2705,6137,3787,3919,6758,7323,7260)$ \\
\hline “ & 30 & 2 & 239005 & $(1601,4313,2907,2602,4760,4736,4199)$ \\
\hline A2003301.1825 & 27 & 8 & 175677 & $(3043,6458,3950,4307,7070,7751,7914)$ \\
\hline “ & 27 & 1 & 427904 & $(1917,4583,3240,3040,5013,5043,4599)$ \\
\hline 4 & 23 & $34(\mathrm{C}, \mathrm{Sm})$ & 29285 & $(2641,5345,4687,4405,5351,4883,4249)$ \\
\hline " & 8 & $35(\mathrm{Sm})$ & 9662 & $(2539,4716,4978,4547,4572,4448,4058)$ \\
\hline A2003322.1845 & 32 & 2 & 359486 & $(1268,3177,2413,2091,3418,3383,3000)$ \\
\hline “ & 32 & 1 & 244095 & $(2104,4758,3060,3074,5266,5768,5672)$ \\
\hline “ & 29 & $52(\mathrm{Sn})$ & 1644 & $(6720,10762,10806,11117,4749,1633,1308)$ \\
\hline “ & 23 & $55(\mathrm{Sn})$ & 1085 & $(4141,7934,6932,6859,4188,1405,833)$ \\
\hline A2004032.1825 & 30 & 7 & 253813 & $(3116,6424,4859,4828,6088,5784,5536)$ \\
\hline “ & 30 & 1 & 349768 & $(1577,3750,2686,2461,4086,4319,4189)$ \\
\hline “ & 29 & $26(C \& S n)$ & 52376 & $(4365,8354,7933,7388,7600,6190,4493)$ \\
\hline A2004080.1825 & 30 & 10 & 118451 & $(4329,8842,5819,6268,9027,9248,9580)$ \\
\hline " & 30 & 1 & 485130 & $(2373,6039,3890,3771,6091,6102,5778)$ \\
\hline “ & 29 & $44(\mathrm{Sn})$ & 2432 & $(9973,14467,16929,16901,4030,1362,1203)$ \\
\hline “ & 26 & $48(\mathrm{C})$ & 2112 & $(8008,14955,13442,13241,13642,13274,11868)$ \\
\hline “ & 23 & $45(C ?)$ & 5102 & $(7332,13058,12133,11934,11438,6707,4862)$ \\
\hline A2004163.1855 & 35 & 8 & 101772 & $(4430,9296,5457,6018,9927,11217,12042)$ \\
\hline “ & 35 & 1 & 501809 & $(2451,6164,3818,3662,6660,6964,6569)$ \\
\hline & 30 & $56(\mathrm{C})$ & 1958 & $(6933,13305,11376,11191,12432,11805,11084)$ \\
\hline & 29 & $55(\mathrm{C})$ & 1599 & $(6140,9656,9553,9975,5226,4584,4909)$ \\
\hline A2004268.1850 & 28 & 5 & 250338 & $(3217,7058,4150,4403,7611,8382,8567)$ \\
\hline “ & 28 & 1 & 352494 & $(1805,4683,2978,2747,5053,5210,4808)$ \\
\hline “ & 23 & $49(\mathrm{C} ?)$ & 3726 & $(5595,10482,9488,9001,9633,6548,4921)$ \\
\hline “ & 23 & $59(\mathrm{C} ?)$ & 1712 & $(6244,11798,10277,10030,11027,10279,9595)$ \\
\hline A2004334.1835 & 30 & 8 & 152117 & $(2706,5740,3711,3862,5557,5842,6243)$ \\
\hline “ & 30 & 1 & 451464 & $(1361,3530,2435,2180,3569,3492,3264)$ \\
\hline “ & 29 & $31(\mathrm{Sn})$ & 14318 & $(5113,9262,8352,8203,5405,2239,1687)$ \\
\hline “ & 26 & $44(C ?)$ & $\overline{4283}$ & $(3954,7561,6455,6264,6644,6025,6062)$ \\
\hline A2005059.1820 & 31 & 15 & 76389 & $(5465,11014,9041,8659,9708,8479,7105)$ \\
\hline “ & 31 & 1 & 352354 & $(1992,5715,3425,3209,5538,5262,4685)$ \\
\hline “ & 30 & $33(\mathrm{C})$ & 23399 & $(8000,14658,13619,12899,12160,9313,6447)$ \\
\hline
\end{tabular}

